Hepatitis C prevention and convenience: Why do people who inject drugs in sexual partnerships 'run out' of sterile equipment?

\title{
Authors:
}

*Professor Suzanne Fraser

National Drug Research Institute

Faculty of Health Sciences

Curtin University, Australia

Mr Jake Rance

Centre for Social Research in Health

Faculty of Arts and Social Sciences

University of New South Wales, Australia

Professor Carla Treloar

Centre for Social Research in Health

Faculty of Arts and Social Sciences

University of New South Wales, Australia

${ }^{*}$ Corresponding author 


\section{Hepatitis C prevention and convenience: Why do people who inject drugs in sexual partnerships 'run out' of sterile equipment?}

\section{Abstract}

Rates of hepatitis $\mathrm{C}$ virus transmission among people who inject drugs in Australia remain high despite decades of prevention education. A key site of transmission is the sharing of injecting equipment within sexual partnerships. Responsibility for avoiding transmission has long been understood individually, as have the measures designed to help individuals fulfil this responsibility, such as the distribution of sterile injecting equipment. This individualising tendency has been criticised for placing an unfair level of responsibility on poorly resourced, marginalised people, and ignoring the social nature of injecting drug use and related health care. Likewise, although research has demonstrated that injecting drug use is gendered, gender and sexual partnerships remain marginal to health promotion efforts. In this article we address these weaknesses, drawing on a qualitative, interview-based project that explored equipment sharing within (hetero)sexual partnerships. In conducting our analysis we explore a key theme that emerged in discussions about accessing and sharing injecting equipment, that of convenience, using critical marketing theory to understand this theme. In particular we investigate the issues of convenience that affect the use of sterile injecting equipment, the many factors that shape convenience itself, and the aspects of equipment use that go beyond convenience and into the realm of intimacy and meaning. We conclude that injecting equipment needs to be both meaningful and convenient if sharing within partnerships is to be reduced further. 


\section{Hepatitis C prevention and convenience: Why do people who inject drugs in sexual partnerships 'run out' of sterile equipment?}

Rates of hepatitis $\mathrm{C}$ virus transmission among people who inject drugs in Australia appear to be decreasing but remain high (Razali, Amin, Dore, Law \& The HCV Projections Working Group, 2009; The Kirby Institute, 2014). A key site of transmission is the sharing of injecting equipment within sexual partnerships. Responsibility for avoiding transmission has long been understood individually, as have the measures designed to help individuals fulfil this responsibility, such as the distribution of sterile injecting equipment. This individualising tendency has been criticised for placing an unfair level of responsibility on poorly resourced, marginalised people, and ignoring the social nature of injecting drug use and related health care (Fraser, 2004; Fraser, 2010). Likewise, although research has demonstrated that injecting drug use is gendered, gender and sexual partnerships remain marginal to health promotion efforts (Dwyer, Fraser, Treloar, 2011). Prevention education materials continue to treat readers as sole gender-neutral agents operating in an environment in which other people are to be seen only as a source of infection (Dwyer, Fraser, Treloar, 2011; Fraser, 2004). The packaging and distribution of 'fitpacks' to reduce injecting equipment sharing also tend to treat the target audience as a population of atomised gender-neutral individuals, each of whom should be supplied with individualised units of injecting equipment. In this article we ask, how can the socially embedded character of injecting within gendered sexual relationships be better acknowledged and accommodated in efforts to limit the spread of hepatitis C?

One way to do this is to investigate equipment sharing via a research method that sees partnerships instead of individuals as the primary unit of analysis. This is the purpose of the project on which this article is based, an Australian National Health and Medical Councilfunded project entitled 'Understanding and prevention hepatitis $C$ transmission in sexual partnerships'. In conducting our analysis we explore a key theme that emerged in 
discussions about accessing and sharing injecting equipment in sexual partnerships, that of convenience, drawing on recent critical marketing theory to understand this theme. We begin with a background section, then follow this by detailing our approach and methods. In the analysis section that follows we explore the ways in which convenience affects the use of sterile injecting equipment, the many factors that shape convenience, and the issues the interviews illuminate that go beyond convenience and into the realm of intimacy and meaning. We conclude by arguing that people who inject drugs are consumers and warrant understanding and servicing on the same terms as other consumers. We further conclude that, as consumer objects, fitpacks may need to be both meaningful and convenient if sharing within partnerships is to be reduced further.

\section{Background}

In Australia an estimated 10,000 new infections occur each year, with nearly $90 \%$ of these among people who inject drugs (Razali et al., 2007). Over the past decade around one in six people who inject drugs who participated in the annual Australian Needle Syringe Programme Survey reported recent receptive syringe sharing (Iversen, Chow, \& Maher, 2014). In 2013, injecting drug use surveillance data indicated that approximately $42 \%$ of needle-sharing incidents occurred between regular sexual partners (Iversen et al., 2014). Similar patterns, if somewhat higher rates, were found in earlier studies. Cao and Treloar (2006) found $64 \%$ of participants who reported needle-sharing claimed they done so with their partner; while in another study, more than half $(51.3 \%)$ of sexual partners surveyed acknowledged sharing needles with each other (Bryant, Brener, Hull, \& Treloar, 2010). This sharing between sexual partners has significant implications for the transmission of hepatitis C.

Sexual relationships frequently incorporate a high degree of intimacy, collaboration and sharing. This is as much the case for partnerships between people who inject drugs as for other partnerships. Sexual relationships are qualitatively different from other relationships 
and as such warrant specific investigation so that prevention education can best respond to their particular features and effects. Despite the relevance of sexual partnerships to hepatitis C prevention or transmission, very little research to date has focused on them (El-Bassel, Shaw, Dasgupta, \& Strathdee, 2014; Rhodes \& Quirk, 1998; Seear et al., 2012; Simmons \& Singer, 2006). Avoiding transmission continues to be understood as predominantly an individual responsibility (Dwyer, Fraser, \& Treloar, 2011; Fraser, 2004; Fraser, Treloar, Bryant \& Rhodes, 2014). This individualisation is reflected and reinforced not only through the prevention education and health promotion materials produced for people who inject drugs (Dwyer et al., 2011; Fraser et al., 2014) but the practical measures designed to enable the fulfilment of this responsibility, such as the distribution of sterile injecting equipment. An 'atomistic' approach to education and prevention is perhaps not surprising in that it characterises public health understandings of illicit drug use and dependence as a whole. As Simmons and Singer (2006, p. 17) argue:

illicit drug dependency and addiction tends to be seen and treated as an individual problem, as if drug users were not capable of having romantic partnerships, and certainly not romantic partnerships that are supportive and caring.

In this article we take a different approach to this issue, considering the public health dimensions of drug use and hepatitis $C$ transmission via the collective agencies of markets and partnerships.

In Australia, the majority of needle syringes used by people who inject drugs are distributed via government-funded needles syringe programmes (NSPs) (The Kirby Institute, 2014). In this way, responsible individuals may access specific tools to manage risk. The primary function of NSPs is to provide sterile injecting and ancillary equipment (alcohol swabs, sterile water and so on), along with a means of safe disposal. They also supply information on safer injecting and referrals to other services (The Kirby Institute, 2014) as well as safe sex information and products, such as condoms, to help prevent the sexual transmission of blood borne virus and other sexually transmitted infections (Health Outcomes International 
Pty Ltd, National Centre in HIV Epidemiology and Clinical Research \& Drummond, 2002). Automatic dispensing machines dispense needle syringes and ancillary equipment, providing additional coverage during the hours staffed outlets are unavailable, and some community pharmacies provide equipment for sale or for free on exchange of used equipment (Bryant, Topp, et al., 2010; Islam, Wodak, \& Conigrave, 2008). While this suite of services constitutes a significant contribution to hepatitis $C$ prevention in Australia, it has not achieved the desired reduction in transmission. Research suggests that coverage remains an issue. Indeed, estimates of coverage indicate that $20 \%$ of injections in Australia are not supported by sterile equipment (Iversen et al., 2012). However, other research has shown coverage rates are not directly associated with equipment sharing (Bryant et al., 2012). Bryant and colleagues argue that once coverage has reached a certain threshold, other factors beyond equipment distribution affect how equipment is used. In this article we set aside the question of coverage to focus on the 'other factors' that can shape equipment use.

\section{Approach}

In analysing the data collected for this project we draw on contemporary critical approaches to public health. As already noted, individualising approaches to public health interventions, including those in the field of alcohol and other drug use, have been widely criticised. As Cameron Duff puts it in a new book asking us to think of health not as a fixed state located within the individual subject but as an assemblage of forces and objects,

Among a panoply of forces, the subject [for instance, the individual drug user] is picked out merely because it is the most familiar, the one considered most amenable to intervention if not transformation. (Duff, 2014, p. 142-3)

As we have noted, the project on which this article is based asks what happens if we resist this familiar path, focusing instead on partnerships and on objects and their circulation. Our analysis will also draw on the field of marketing, in particular on a key idea that can be seen as animating our participants' discussions of sharing and equipment access: convenience. 
While we do not argue that the discipline of marketing understands social issues such as injecting better than others, we consider elements of marketing theory, especially those produced in a critical engagement with traditional marketing theory, useful for shedding new light on matters that might otherwise be taken for granted.

In 1992 Stephen Brown argued that marketing had much to gain from the emerging theoretical terrain of poststructuralism. Casting a critical eye over marketing's traditional compliance with Enlightenment assumptions about 'ineluctable progress, scientific achievement and freedom' he traced the rise of postmodernism's idealisation in art of pastiche, eclecticism and play, and its contribution to changing ideas in science that saw objectivity criticised as a fantasy, and indeterminacy and contingency proposed as guiding principles in the advent of chaos theory, fractal geometry and so on. This shifting ground, he argued, had serious implications for traditional marketing. Making generalisations about consumers and products, for example, or establishing universal truths about the market, were no longer plausible goals. Instead marketing needed to embrace the fundamental questions these developments raised about the subjects of marketing, and the utility of narrow, rigid models such as marketing's 'product life cycle' (Levitt, 1965: development, introduction, growth, maturity, decline) and Maslow's (1954) psychological 'hierarchy of needs' (physiological, safety, love/belonging, esteem, self-actualisation).

Of especial relevance for our purposes, these developments redirected attention away from generalisations to 'the uniqueness, diversity, plurality and idiosyncrasy of each and every individual' (Brown, 1992, p. 26). This plurality and idiosyncrasy is particularly significant for the notion of convenience. As Elizabeth Shove (2003, p. 416) puts it, in trying to understand how we use everyday objects (such as injecting equipment),

What is required is an understanding of how such elements are integrated into systems of provision within and beyond the home, how they are fitted into 
constantly shifting frameworks of 'normality', and how concepts of service are thereby reconstructed.

Here goods and services can be understood less as stable phenomena for which markets must be found and more as the effects of particular systems of provision (such as the traditional system in the West in which budget management and household shopping are the duty of the 'housewife') and shifting frameworks of the normal (such as the movement of women into the workforce). This kind of inquiry is important because it locates the use of objects not only in the intentional responsibilised hands of individuals but also in changeable, open social and economic processes such as the postwar rise of feminism and the concomitant economic restructuring towards dual-income households (that reshaped incomes, and shopping and spending patterns).

Shove and others also talk about the composition and nature of convenience, an idea often taken for granted but one well worth unpacking. Farquhar and Rowley (2009, p. 434) offer the following definition of convenience:

a judgment made by consumers according to their sense of control over the management, utilisation and conversion of their time and effort into achieving their goals ...convenience is not an inherent characteristic of a service [or object]. [Instead it must be understood in context.]

Writing much earlier, Yale and Venkatesh (1986) make the related and still relevant point that convenience has numerous dimensions and argue that marketing needs to understand them to achieve convenience. The dimensions they nominate are: time utilisation, accessibility, handiness, appropriateness, portability and avoidance of unpleasantness. While they are useful pointers to the complexity of convenience, and are listed here to illustrate this complexity, they do not all equally apply to the material analysed here. We consider the concept of complexity heavily dependent upon specific social conditions and practices and its dimensions highly variable. To consider for a moment the relevance of this approach, however, we sometimes assume that NSPs deliver convenience, and that where 
there are gaps, vending machines could fill them. This is certainly part of the picture (Cama et al., 2014), but as this theory proposes (and as our interviews, discussed later, also suggest) convenience is more complicated than this. In addition, convenience, especially if narrowly defined, is not always the main priority for consumers.

Taken together, these insights about the multiple nature of markets, the many dimension of convenience, the place of factors other than convenience in shaping consumption, and the relationship between all these elements and broad social 'frameworks of normality', form the basis for our analysis to follow.

\section{Methods}

The data set on which this analysis is based comprises 80 in-depth qualitative interviews conducted with individuals in heterosexual partnerships where injecting drug use occurred. The project focused on heterosexual partnerships because this is where the vast majority of partnership injecting occurs (Iversen et al., 2014). This is not to suggest injecting drug use among same sex couples should be ignored: indeed it warrants standalone research in which the unique dynamics of power and gender can be comprehensively examined.

Participants were recruited during 2012 and 2013 from Australia's two most populous states, New South Wales (NSW) and Victoria. Purposive recruitment was used to reach couples in which both partners identified as people who inject drugs (PWID). Recruitment and interviewing took place at four inner-city harm reduction services: a needle syringe program (NSP) and a harm reduction service in NSW, and two primary healthcare centres in Victoria. Staff from the four recruitment sites alerted service users to the study. The study was approved by the Human Research Ethics Committee of The University of New South Wales (reference $\mathrm{HC12430)}$ and from the relevant human research ethics committees at each site. Written informed consent was obtained from all participants. 
Partner research takes a range of forms (Eisikovits \& Koren, 2010). In our study both members of the partnership were interviewed separately by the same researcher, and the importance of confidentiality was reiterated to all participants. Interviews followed a semistructured format. The interview guides were organised around core themes of injecting drug use, hepatitis $C$ and intimate sexual partnerships. Participants were asked to describe the nature of their current relationships (including any involvement with other sexual partners), their knowledge of hepatitis $\mathrm{C}$ and its relevance to the relationship, their experiences injecting with partners and friends, including equipment sharing, and their experiences with harm reduction services. Interviews were between 30 and 60 minutes in duration. Each participant was reimbursed $\$ 30$ to cover time and travel expenses.

The complete dataset comprised 34 partnerships and 12 'sole' participants, with equal numbers of men and women $(n=40)$. Ages ranged from 19 to 61 years. 'Sole' participants were included on the basis of having had relationship experience (current or prior) involving injecting drug use. In total, then, we secured 75 accounts of partnerships currently injecting drugs (involving accounts from 41 current couples: 34 where both members of the partnership were interviewed and 7 where only one partner participated) along with a further 5 accounts from sole participants (currently either single or in a relationship with someone who does not inject drugs) who reflected on prior experiences of partnerships where injecting drug use had occurred. Of these 41 couples, 29 reported sharing within the partnership. Such events were nonetheless characterised by participants as atypical: a 'last resort'. Only one participant reported recently sharing with someone in addition to their partner. $^{1}$

The interviews were digitally recorded, transcribed verbatim and de-identified to ensure anonymity. Each participant was given a pseudonym to preserve anonymity. The research team then collaborated on a coding frame. Our approach to data coding was also informed 
by a partnership-related pilot study undertaken by the research team in 2011 and 2012, and by our knowledge of the existing literature. The transcripts were then entered into a qualitative data management program, NVivo 9. Codes were applied by one researcher (JR) and summaries of nodes reviewed by all authors to assist identifying concepts and support emerging hypotheses. This article analyses the node that collated all interview material on sharing within partnerships. It presents extracts form this node, noting the age group of the participant, the location of the interview (New South Wales or Victoria), the sex of the participant, and the hepatitis $\mathrm{C}$ serostatus reported by the participant.

\section{Analysis}

In commencing this analysis we begin by noting the value of treating people who inject drugs like other consumers of goods and services, as knowledgeable and reasonable members of the community whose desires and priorities cannot simply be dismissed as dysfunctional or disordered. Related to this, we recognise that our participants are affected by the same issues as other consumers, and that these issues help shape their decisions about accessing healthcare and related goods and services. Viewed from this perspective, our first finding is unsurprising. When asked about sharing injecting equipment and invited to explain the reasons for the occasional sharing they described, many participants reported simply 'running out' of new equipment. This occurred even though knowledge of hepatitis $\mathrm{C}$ risks and routes of transmission was generally good. Of course, running out of staples happens to all consumers from time to time, no matter how important these products are. In our interviewing and data analysis we tried to tease out the circumstances and thinking surrounding this running out, finding a number of overlapping issues to do with convenience, the complexities of convenience, and questions beyond convenience (to do with relationships, intimacy and other aspects of meaning). In the two sections that follow we first consider the issues participants raise that explicitly relate to convenience (and indicate the 
complexity of convenience), and second we consider other relevant meanings associated with injecting and equipment sharing in partnerships.

\section{Convenience}

As already noted, our interview participants were asked whether they ever shared injecting equipment with sexual partners, and if so, why. Very often they replied that they did so only rarely, and when they did it was because they had simply 'run out' of new equipment. Rather than take this explanation at face value, we explored the issues behind, and circumstances surrounding, running out. When we did so, the picture became much more complex. Some participants reported collecting large supplies of equipment from NSPs and then distributing their stock to others in need. Others said they trusted their partners, so sharing was not considered very risky so that vigilance about supplies was not always a top priority. Others likened injecting equipment to the most mundane of household objects (in one case it was described as like bread or milk), and presented running out as an effect of this banality and forgettability. Embedded in all these accounts was also the sense that running out of equipment is not a good thing - that new equipment is always preferable where convenient.

What can make or break this convenience? A wide range of things. Many participants referred to picking up boxes of 100 needle-syringes from NSPs as a way of enhancing convenience. Brian for instance explained picking up boxes of hundreds saying:

We usually only come in once or twice a month, just not wanting to come in to the city. (20s, NSW, M, neg.)

Elias also described picking up hundreds, saying that sharing only happened when these ran out and accessing more equipment was not possible at the time.

[Bulk boxes] make us feel a lot safer. We know we're going to have one each for at least 20 days...almost the month...but there's moments like Christmas Day and Boxing Day you run short...(20s, NSW, M, neg.) 
So here opening hours and public holidays shape convenience, undermining some of the convenience built up by storing large numbers of needle-syringes. But other factors also shape convenience. Elias also added that:

it's mostly when we're on ice [that we share]... because you know we're up all the time and things aren't up all the time... (20s, NSW, M, neg.)

Here, NSP opening hours can be fundamental to convenience, but not always.

Overall these comments suggest that use of new injecting equipment is in some cases shaped by convenience of access according to NSP opening hours and location. This could be seen as fundamentally a matter of coverage, which is undoubtedly a key issue for prevention (Cama et al., 2014). But convenience is more than this suggests. As Yale and Venkatesh (1986) argue, convenience comprises a range of features and considerations. These vary because the market itself is diverse, and cannot be treated as a homogenous group. For Brian, travelling from the suburbs into the city means regular opening hours and location are key considerations. For others, such as Clare who injects methadone, and her partner Cliff, who injects heroin, having access to the right sort of equipment (needles of the appropriate gauge and length for femoral-vein injecting) was the primary concern:

[B]ecause I go in my groin I need the big green needle ... [Cliff] still uses a one mil fit [because he] still uses gear, I don't. (40s, NSW, F, pos.) Homeless participants reported additional challenges in addition to access to injecting equipment: portability and detectability. For them, transporting injecting supplies without attracting attention from police took priority. As Cath explains:

I could get [a box of] 100 if I wanted to, but it's just storing them. I don't want to be walking around the streets with 100 needles, because then l'll get pulled over and the police are like 'what the fuck?' ... (30s, Vic, F, neg.)

While Cath describes limiting her stock of equipment to what she can carry discreetly, another homeless participant, Christine, explains a different approach: 
Stash them ... you pick the right spot, like places no one wants to go. (20s, NSW, F, pos.)

Thus, while coverage might seem likely to address the need for convenience, for many it would not necessarily do so, at least not on its own. Also relevant are the 'effects of particular systems of provision' (Shove, 2003, p. 26) of objects, here the supply of injecting equipment in a legal environment shaped by prohibition.

The other issue positioned alongside the complexity of convenience is the perhaps obvious one that convenience is not always the key focus for consumers. Green consumerism is a case in point explored in the marketing literature (Gehrt and Yale, 1993; Moisander, 2007) will we recycle now that it is convenient enough to do so? Will we ride bikes if they're left for hire on every street corner? Clearly, convenience is not always enough to shape conduct. For Seth, as for a number of other participants who occasionally ran out of equipment, an ethos of community care also shaped the choices he made about collecting injecting equipment:

we might take a box of a hundred needles because we know that ... people come by our house at whatever time it is [saying] 'oh I need a freshy' [sterile needle-syringe]. At least you can give them clean equipment, and [if] it stops one person from passing on a blood-borne virus then so be it. (30s, NSW, M, pos.)

Likewise, Jenn explains:

... sometimes we give them to people, like, we live in a housing commission and people sometimes come knocking and ask for fits, which is... but you know l'm happy to [give them away] because I know that l've got an infection in my veins and I know, I wouldn't want people to, I got it from re-using my needles and it nearly killed me, so I'm happy to give to friends or whatever, you know. It's just so important to have clean fits. (30s, Vic, F, pos.) 
Our data suggest that many participants routinely collected, distributed and disposed of large quantities of injecting equipment for friends, neighbours and their broader injecting communities. They explain that these practices of care could at times leave them without new equipment. Participants also describe other practices of care and responsibility that could lead to the running out we describe. Jim (hepatitis C [HCV]-positive) describes breaking the needles off his used syringes to ensure others do not reuse the equipment. As he explains, however, this can also mean that where he does not have any new equipment, he is unable to re-use his own. This leaves him vulnerable to re-using someone else's:

I think being really cautious and really careful can come back to bite you in the ass ... Because not only do I bin mine but I snap the end off and put the piece that could hurt anyone down the barrel and jam the barrel down there so it is never going to hurt anybody ... (60s, NSW, M, pos.)

Collecting large volumes of injecting equipment to hand out to neighbours and friends; breaking off tips to put syringes out of action, then borrowing other people's when you finally run out of new ones (also Jack: M, 20s, Vic): these are examples of running out that do not fit into simple explanations of insufficient coverage, or of personal convenience. In signalling the place of care for others in daily life, they also indicate another, broader, issue: that although cheap and disposable, injecting equipment is laden with meaning. This is the next area we wish to consider in this analysis of running out. As we will see, it is one that often directly relates to intimate partnerships.

\section{Meaning}

As the marketing literature on convenience suggests, goods and services carry important meanings that shape practice beyond whether they are quick to use or easy to access (Gehrt and Yale, 1993). Our data contain a range of examples of this, especially of the meanings at work in sexual partnerships. For example, some participants detail the care they or their partners take when supplies of new equipment run so low they do not have 
enough for each person. These examples are especially important in demonstrating the meaning attached to injecting equipment and in raising the possibility that convenience is not always the sole consideration for consumers. As Pam explains,

[E]very now and then, when we have been stuck and we've only got sort of like one fit between us ... he'll [partner, Patrick] make sure that I use it ... then he'll rinse it and use it $\ldots$ because he has it [HCV] and I don't. (50s, NSW, F, neg.) Here Pam describes an arrangement in which her partner demonstrates care for her through the order in which they share equipment. She always goes first because, as far as they are both aware, she is HCV-negative and he is HCV-positive. As Patrick explains himself, 'I look after myself and I look after my loved ones.' Another participant, Tanya, describes a similar process in which care is communicated via the sharing of injecting equipment. In this case, however, her partner is HCV-negative while she is HCV-positive, and the caring behaviour is framed specifically through a traditional gendered notion of chivalry:

There have been a grand total of three or four times when we've had to share ...we've never shared a dirty one ... [but] we've shared a clean one. Both me and him [partner, Tim] agree that ... I would go first, he would go second ... Ladies first. (20s, NSW, F, pos.)

In the parallel interview conducted with Tanya's partner Tim, he reports insisting on taking second place despite the risk of transmission because this is a signal of gentlemanly behaviour. Indeed, he also describes injecting Tanya's blood on one occasion in an effort to seroconvert and share her predicament. Thus, alongside the more complicated understandings of convenience we have identified and of what makes accessing and using sterile injecting equipment manageable and worthwhile to consumers, other issues of meaning and practice must be considered: what this equipment signifies and communicates - how it functions socially in partnerships, and how these functions relate to (gendered) 'frameworks of the normal'. For some participants, such as Patrick and Tim (and Tanya and Pam too) the way equipment is shared or not shared works to communicate love and trust. Within these two partnerships, it seems, injecting equipment fulfils a number of functions 
beyond the strictly utilitarian. It allows Patrick and Tim to express their love and commitment to their partners, in Patrick's case via a deeply held ethic of care towards others, and in Tim's via the (traditional chivalric) masculinity he cherishes (he explains earlier in the interview) as a reflection of his mother's early influence.

While for a number of couples within our dataset the meanings and practices associated with injecting equipment reflected love and trust, for a minority the shared injecting process signified something quite different. For Mandy (40s, VIC, pos.), her partner Mike's (30s, VIC, pos) impatient insistence on injecting as soon as they acquired their drugs - invariably in public and with the minimum of care or hygiene - had become a source of distress and defeatism.

[I]t's pretty scary sometimes what I see ... he [Mike] does the mixing of the dope... [W]e never go home and have it; whereas if I had it my way, we'd be taking the drugs home, and it would be a whole lot cleaner... [I]t's got the point where l've just given up. It's like I think, 'Well l've got hepatitis C now.'

Rachel (40s, VIC, pos.) describes her drug use with partner Robert (40s, VIC, pos.) similarly. She, like Mandy, describes distress in the face of her partner's impatience and control over the injecting process.

[H]e can't wait to go home and have it. We'll stop in a laneway or whatever and have it. I get really paranoid but he loads mine up and I have it anyway ... We do fight a lot while we're on drugs.

In all these cases injecting equipment and how it is used has meaning beyond utility and convenience per se. This meaning emerges at least in part from the systems that govern provisioning, here the political and legal context that prohibits certain kinds of drug consumption and thereby institutes particular drug use risks and deprivations. Where such risks and deprivations emerge to be addressed, the manner in which they are addressed is 
informed, as Shove (2003) puts it, by 'shifting frameworks of normality' including gendered modes of care such as chivalry.

As outlined in an earlier article published from the project drawn on here (Fraser, 2013), we plan to address the important issue of the meanings attached to injecting equipment further by exploring new partnership-oriented fitpack designs and specifically tailored hepatitis $C$ prevention messages. These could acknowledge and integrate the partnerships people who inject drugs value as well as make safe injecting easy. As Vitellone (2003) argues, apparently mundane objects such as needle-syringes can play a formative role in social identities and relationships. Here we are looking a long way past the language of 'running out' as a simple failure of memory or access and the solution of coverage it implies.

\section{Conclusion}

As noted at the outset, sharing injecting equipment has been identified as an important issue for hepatitis $C$ prevention, but the reasons for sharing are not yet as well understood as they might be. In our study many participants gave running out of equipment as a key reason for sharing. Here we have interpreted this issue through the idea of convenience, a concept that implicitly informs calls for increasing NSP coverage, but which also offers a range of other insights that can be used to understand equipment access and use. Drawing on marketing theory such as Yale and Venkatesh's (1986) work on the many dimensions of convenience, we identified a way of framing convenience that helped draw attention to the multiple factors that make up convenient access to new injecting equipment. In addition, we used the theory to observe that patterns of consumption emerge within shifting social and economic conditions (including gender and other 'frameworks of the normal' such as Australia's legal frameworks that inform personal storage and transportation practices as well as 'systems of provisioning' such as the NSP system), and to observe that at times consumers rank convenience below other values, even for apparently quotidian products. While the 
importance of NSP coverage should not be underestimated, other issues to do with access also need to be taken into account when thinking about how to design services and measures to reduce transmission.

We would also argue that the status of injecting equipment as everyday, simultaneously banal and part of the fabric of who we are and how we understand ourselves, suggests there may be benefits to rethinking its presentation. Rather than focusing solely on individual units (and individual consumers) and treating equipment as largely 'blank' or absent of meaning, we might find it productive to recognise the meaning and value it accumulates, and the 'marketplace' of priorities and options NSPs exist within, and work with this to enhance particular meanings and practices. This could in turn interrupt the somewhat naturalised process of running out reported in our research. Injecting equipment might, it seems, need to be both meaningful and convenient if sharing within partnerships is to be reduced further.

Acknowledgments: The research project reported on here was funded by the National Health and Medical Research Council (Australia). The authors report no conflict of interest. The National Drug Research Institute at Curtin University is supported by funding from the Australian Government under the Substance Misuse Prevention and Service Improvement Grants Fund. The first author (SF) is supported by an Australian Research Council Future Fellowship (FT120100215). The Centre for Social Research in Health is supported by a grant from the Australian Government Department of Health. The authors thank the participants in this study and the anonymous reviewers for $\mathrm{CPH}$. 


\section{References}

Bryant, J., Topp, L., Hopwood, M., Iversen, J., Treloar, C., \& Maher, L. (2010). Is point of access to needles and syringes related to needle sharing? Comparing data collected from pharmacies and needle and syringe programs in south-east Sydney. Drug \& Alcohol Review, 29(4), 364-370. doi: 10.1111/j.1465-3362.2009.00154.x

Bryant, J., Brener, L., Hull P. \& Treloar, C. (2010). Needle sharing in sexual relationships: Serodiscordance and the gendered character of injecting. Drug \& Alcohol Dependence, 107, 182-7. doi: 10.1016/j.drugalcdep.2009.10.007

Bryant, J., Paquette, D. \& Wilson, H. (2012). Syringe coverage in an Australian setting: Does a high level of syringe coverage moderate syringe sharing behaviour? AIDS \& Behavior, 16(5), 1156-1163. doi: 10.1007/s10461-011-0010-3

Cama, E., Brener, L. \& Bryant, J. (2014). Characteristics and attendance patterns of a fixed site NSP and nearby SVM: Benefits of 24-hour access to sterile injecting equipment. Drugs: Education, Prevention \& Policy, 476-481. doi: 10.3109/09687637.2014.956051

Cao, W. \& Treloar, C. (2006). Comparison of Needle and Syringe Programme attendees and non-attendees from a high drug using area in Sydney, New South Wales. Drug and Alcohol Review, 25, 439-44. Retrieved from:

http://informahealthcare.com/doi/abs/10.1080/09595230600891282

Duff, C. (2014). Assemblages of Health, Dordrecht: Springer.

Dwyer, R., Fraser, S. \& Treloar, C. (2011). Doing things together? Analysis of health education materials to inform hepatitis $C$ prevention among couples. Addiction Research \& Theory, 19(4), 352-361. doi: 10.3109/16066359.2011.562619

Eisikovits, Z. \& Koren, C. (2010). Approaches to and outcomes of dyadic Interview analysis. Qualitative Health Research, 20(12), 1642-1655. doi: 10.1177/1049732310376520

El-Bassel, N., Shaw, S. A., Dasgupta, A., \& Strathdee, S. A. (2014). People who inject drugs in intimate relationships: it takes two to combat HIV. Curr HIV/AIDS Rep, 11(1), 45-51. doi: $10.1007 / \mathrm{s} 11904-013-0192-6$

Farquhar, J. \& Rowley, J. (2009). Convenience: A services perspective. Marketing Theory, 9, 425-438. doi: 10.1177/1470593109346894

Fraser S. (2004). 'It's Your Life!': Injecting drug users, individual responsibility and hepatitis C prevention. Health 8(2), 199-221. doi: 10.1177/1363459304041070

Fraser, S. (2010). Hepatitis C and the limits of medicalisation and biological citizenship for people who inject drugs. Addiction Research \& Theory, 18(5), 544-556. doi: 10.3109/16066350903497551

Fraser, S., Treloar, C., Bryant, J. \& Rhodes, T. (2014). Hepatitis C health promotion needs to be grounded in social relationships. Drugs: Education, Prevention \& Policy, 21(1), 88-92. doi: 10.3109/09687637.2013.776517 
Fraser, S. (2013). The missing mass of morality: A new fitpack design for hepatitis C prevention in sexual partnerships. International Journal of Drug Policy, 24, 212-219. doi: 10.1016/j.drugpo.2013.03.009

Gehrt, K. \& Yale, L. (1993). The dimensionality of the convenience phenomenon: A qualitative re-examination. Journal of Business and Psychology, 8(2), 163-180. doi: 10.1007/BF02230383

Health Outcomes International Pty Ltd, National Centre in HIV Epidemiology and Clinical Research, \& Drummond, M. (2002). Return on investment in needle and syringe programs in Australia: Summary Report. Canberra: Commonwealth Department of Health and Ageing.

Islam, M., Wodak, A., \& Conigrave, K. M. (2008). The effectiveness and safety of syringe vending machines as a component of needle syringe programmes in community settings. [Review]. International Journal of Drug Policy, 19(6), 436-441. doi: 10.1016/j.drugpo.2007.07.006

Iversen, J., Chow, S., \& Maher, L. (2014). Australian Needle and Syringe Program National Data Report 2009-2013. Sydney: The Kirby Institute, UNSW Australia.

Iversen, J., Topp, L., Wand, H. \& Maher, L. (2012). Individual-level syringe coverage among Needle and Syringe Program attendees in Australia. Drug and Alcohol Dependence, 122(3), 195-200. doi: 10.1016/j.drugalcdep.2011.09.030

The Kirby Institute (2014). 2014 Annual Surveillance Report of HIV, viral hepatitis, STIs. University of NSW: Kensington.

Levitt, T. (1965). Exploit the product life cycle. Harvard Business Review, 43(6), 81-94. Retrieved from http://www.ebscohost.com/academic/business-source-complete Maslow, A. (1954). Motivation and personality. New York, NY: Harper.

Moisander, J. (2007). Motivational complexity of green consumerism. International Journal of Consumer Studies, 31, 404-409. doi: 10.1111/j.1470-6431.2007.00586.x

Razali, K., Thein, H., Bell, J., Cooper-Stanbury, M., Dolan, K., Dore, G. \& The HCV Projections Working Group. (2007). Modelling the hepatitis $C$ virus epidemic in Australia. Drug \& Alcohol Dependence, 91(2-3), 228-35. doi: 10.1016/j.drugalcdep.2007.05.026

Razali, K., Amin, J., Dore, G. J., Law, M. G., \& HCV Projections Working Group. (2009). Modelling and calibration of the hepatitis C epidemic in Australia. Stat Methods Med Res, 18(3), 253-270. doi: 10.1177/0962280208094689

Rhodes, T. \& Quirk, A. (1998). Drug users' sexual relationships and the social organisation of risk: The sexual relationship as a site of risk management. Social Science \& Medicine. 2(46), 157-69. doi: 10.1016/S0277-9536(97)00156-1

Seear, K., Gray, R., Fraser, S., Treloar, C., Bryant, J. \& Brener, L. (2012). Rethinking safety and fidelity: The role of love and intimacy in hepatitis $\mathrm{C}$ transmission and prevention. Health Sociology Review, 21(3), 272-286. doi: 10.5172/hesr.2012.21.3.272

Shove, E. (2003). Converging conventions of comfort, cleanliness and convenience. Journal of Consumer Policy, 26, 395-418. doi: 10.1023/A:1026362829781 
Simmons, J. \& Singer, M. (2006). I love you ... and heroin: care and collusion among drugusing couples. Substance Abuse, Treatment and Prevention, 1, 7-19. doi: 10.1186/1747$597 X-1-7$

Vitellone, N. (2003). The syringe as a prosthetic. Body \& Society, 9(3), 37-52. doi: $10.1177 / 1357034 X 030093003$

Yale, L. \& Venkatesh, A. (1986). Toward the construct of convenience in consumer research. In R. J Lutz (Eds.), Advances in Consumer Research (pp. 403-408). Provo: Association for Consumer Research.

\footnotetext{
${ }^{1}$ Nine participants were in part or full-time employment, with nearly all receiving some form of social welfare $(n=71)$ (one participant depended on his partner's income and two participants declined to answer). Over half the participants identified as 'Anglo-Australian' and nearly a quarter as Aboriginal or Torres Strait Islander $(n=17)$. While the majority of the remaining participants identified as having broadly European heritage, $10 \%(n=8)$ comprised a diverse cross-section of ethnicities (Filipino, Armenian, Vietnamese, Indian, Lebanese and Chinese). Serostatus was determined by self-report only and in several cases participants offered conflicting accounts of each other's or their own serostatus. HCV serostatus was fairly evenly shared amongst participants, with 35 reporting to be HCVnegative and $45 \mathrm{HCV}$-positive. Of the dataset's 41 couples, 24 were HCV concordant (11 HCV-negative and 13 HCV-positive) and 17 HCV discordant (10 HCV-positive men and 7 HCV-positive women).
} 